\title{
Design, synthesis and biological evaluation of antimalarial activity of new derivatives of 2,4,6-s-triazine
}

\author{
Mallika Pathak ${ }^{1,2}$, Himanshu Ojha ${ }^{2,3^{*}}$, Anjani K. Tiwari ${ }^{2}$, Deepti Sharma ${ }^{3}$, Manisha Saini ${ }^{1}$ and Rita Kakkar ${ }^{2}$
}

\begin{abstract}
Dihydrofolate reductase (DHFR) is an important enzyme for de novo synthesis of nucleotides in Plasmodium falciparum and it is essential for cell proliferation. DHFR is a well known antimalarial target for drugs like cycloguanil and pyrimethamine which target its inhibition for their pharmacological actions. However, the clinical efficacies of these antimalarial drugs have been compromising due to multiple mutations occurring in DHFR that lead to drug resistance. In this background, we have designed 22 s-triazine compounds using the best five parameters based 3D-QSAR model built by using genetic function approximation. In-silico designed compounds were further filtered to 6 compounds based upon their ADME properties, docking studies and predicted minimum inhibitory concentrations (MIC). Out of 6 compounds, 3 compounds were synthesized in good yield over $95 \%$ and characterized using IR, ${ }^{1} H N M R$, ${ }^{13} \mathrm{CNMR}$ and mass spectroscopic techniques. Parasitemia inhibition assay was used to evaluate the antimalarial activity of s-triazine compounds against 3D7 strain of P. falciparum. All the three compounds $(\mathbf{7}, \mathbf{1 3}$ and $\mathbf{1 8})$ showed 30 times higher potency than cycloguanil (standard drug). It was observed that compound 18 was the most active while the compound $\mathbf{1 3}$ was the least active. On the closer inspection of physicochemical properties and SAR, it was observed that the presence of electron donating groups, number of hydrogen bond formation, lipophilicity of ligands and coulson charge of nitrogen atom present in the triazine ring enhances the DHFR inhibition significantly. This study will contribute to further endeavours of more potent DHFR inhibitors.
\end{abstract}

Keywords: Antimalarial, DHFR inhibitors, Molecular docking, s-Triazine, 3D7 strain

\section{Introduction}

Malaria is a protozoan disease caused by Plasmodium genus. According to WHO report entitled "World malaria report" (2015), 15 countries reported $80 \%$ of cases and $78 \%$ of deaths due to malaria in 2015 [1]. Malaria persists to be one of the critical public health problems in India. Around 1.13 million confirmed cases and 287 deaths were reported in 2015 by the National Vector Borne Disease Control Programme (NVBDCP), out of which $67.1 \%$ was due to Plasmodium falciparum [2]. Odisha, Jharkhand, Chhattisgarh, Madhya Pradesh, Karnataka and north-eastern states except Sikkim,

\footnotetext{
*Correspondence: himanshu.drdo@gmail.com

${ }^{3}$ Division of CBRN Defence, Institute of Nuclear Medicine and Allied

Sciences, DRDO, Timarpur, Delhi 110054, India

Full list of author information is available at the end of the article
}

Maharashtra and Rajasthan are high endemic areas in India.

Antifolate antimalarial drugs such as pyrimethamine and cycloguanil have been used in prevention and treatment of malaria. It is well known that folate metabolism is one of the most studied biochemical pathways of the parasite. Folate metabolism is a critical process being targeted to stop the proliferation of the parasite. The antimalarial activity of therapeutic agents that interfere with folate metabolism has been recognized since long. Two categories of antifolate antimalarial drugs were distinguished by their respective mechanisms of action. In the first category, the sulphonamides and sulphones are chemical analogues of $p$-amino benzoic acid (PABA), an essential precursor for the de novo synthesis of folic acid. The second category includes a variety of drugs that inhibit dihydrofolate reductase (DHFR), the enzyme 
responsible for converting dihydrofolate to the biologically active tetrahydrofolate cofactor [3].

During the earlier trials of chloroguanide as an antimalarial drug on monkeys, rabbits and humans, triazine compounds were identified and isolated. Among these compounds 2-amino-4-( $p$-chloro-anilino)-6,6-dimethyl5,6-dihydro-1,3,5-triazine and $p$-chlorophenylbiguanide were isolated from the urine of monkeys treated with chloroguanide [4]. But both the compounds were found to be inactive against Plasmodium falciparum. However, an isomer of 2-amino-4-( $p$-chloro-anilino)-6,6-dimethyl-5,6-dihydro-1,3,5-triazine,2,4-diamino-5-( $p$ chlorophenyl)-6,6-dimethyl-5,6-dihydro-1,3,5-triazine, isolated from the urine of rabbits [5] and humans [6] were found to be highly active. A large number of dihydrotriazines have been synthesized and many of them showed antimalarial activity [7, 8]. Further, several works have been reported to study the correlation between the structure and the antimalarial activity of triazine compounds. Based on these relationships, several triazine compounds have been synthesized and biologically evaluated for biochemical targets such as polyamine metabolism [9] and DHFR inhibition $[10,11,12]$.

The synthesis of $s$-triazines and their pharmacological applications are well documented [13, 14, 15, 16]. Some $s$-triazine derivatives are reported to possess remarkable antitubercular [17], antimicrobial [18], antibacterial [19] and herbicidal activities [20]. Besides it, $s$-triazine compounds were found to be active as antitumorigenic agents, in chemotherapeutical preparations, active against viruses, protozoa, helminths, pharmacologically effective to treat cardiovascular, neuropsychotic disorders, or inflammatory processes, diuretics, antidiabetic agents, etc. [21].

According to the literature [22, 23] s-triazine compounds fall into the second category that inhibits Plasmodium falciparum-DHFR. DHFR has received considerable attention as it is the target of cycloguanil (a triazine based antimalarial drug) and other antifolates. DHFR is used for prophylaxis and the treatment of Plasmodium falciparum infection [24]. The exponential increase in the emergence of antifolate resistance in Plasmodium falciparum has unfortunately compromised the clinical use of the currently used drugs and therefore highlights the urgent need for new effective antifolate antimalarials $[25,26]$.

During the last two decades, there has been tremendous progress in computational chemistry and Computer Aided Drug Design (CADD). CADD has played a major role in screening of new chemical entities. Under ligand based lead compounds optimization, QSAR study of the bioactive compounds plays a useful role for screening of new potential lead compounds. Therefore, the design of novel chemical entities which can affect selectively the parasite folate metabolism, may lead to discovery of better antimalarial drugs. In our previous reported study we had prepared and discussed 3D QSAR models using Genetic Function Approximation (GFA) method by employing data set of minimum inhibitory concentration (MIC) values of synthetic $s$-triazine compounds tested for DHFR inhibition against cycloguanil resistant strain of Plasmodium falciparum [27]. Using QSAR model no 1 (best model), a number of $s$-triazine compounds were designed by modifying the attached side chains to the carbon atoms of the triazine ring of the parent compound (Table 1).

Under the present study new $s$-triazine compounds were designed and their MIC values were predicted using same QSAR model. The designed compounds were also evaluated for ADME properties and docking score. Based upon these parameters $6 s$-triazine compounds were selected for synthesis. Out of 6 compounds, 3 compounds were synthesized with yield percentage above 95\%. The compounds were characterized using elemental analysis, IR, mass, ${ }^{1} \mathrm{HNMR}$ and ${ }^{13} \mathrm{CNMR}$ experimental techniques. The synthesized compounds were tested against the 3D7 strain of Plasmodium falciparum Rieckmann microassay $[12,16]$. It was observed that all synthesized compounds possessed 30 times higher activity than the standard cycloguanil antimalarial drug.

\section{Materials and methods}

\section{Chemicals and techniques}

All chemicals used in the present study are of analytical grade purchased from Sigma Aldrich and Merck Chemical Company. All the solvents were used after distillation. All the synthesized compounds have been characterized from their analytical, physical and spectral (IR, ${ }^{1} \mathrm{HNMR},{ }^{13} \mathrm{C}$-NMR) data. Infrared spectra (IR) spectra were recorded in $\mathrm{KBr}$ discs on an FT-IR Perkin-Elmer spectrum BX spectrophotometer. ESI-MS spectra were obtained using a VG Biotech Quatrro mass spectrometer equipped with an electrospray ionization source in the mass range of $m / z 100$ to $\mathrm{m} / z$ 1000. ${ }^{1} \mathrm{H}-\mathrm{NMR}$ and ${ }^{13} \mathrm{C}$ NMR spectra were recorded on a Bruker NMR instrument $400 \mathrm{MHz}$ and $100 \mathrm{MHz}$, respectively using $\mathrm{CDCl}_{3}$ and DMSO- $\mathrm{d}_{6}$ as solvents. Elemental analysis was performed on the elemental analyzer Gmbh variable system. All compounds gave satisfactory analytical results.

\section{ADME screening}

QikProp program from Schrödinger Mastero 9.7 [28] was employed to assess the absorption, distribution, metabolism, and excretion (ADME) properties of the compounds. QikProp predicts both pharmaceutically significant descriptors and physically relevant properties. 
Table 1 Structural features of the designed inhibitors and predicted pMIC values

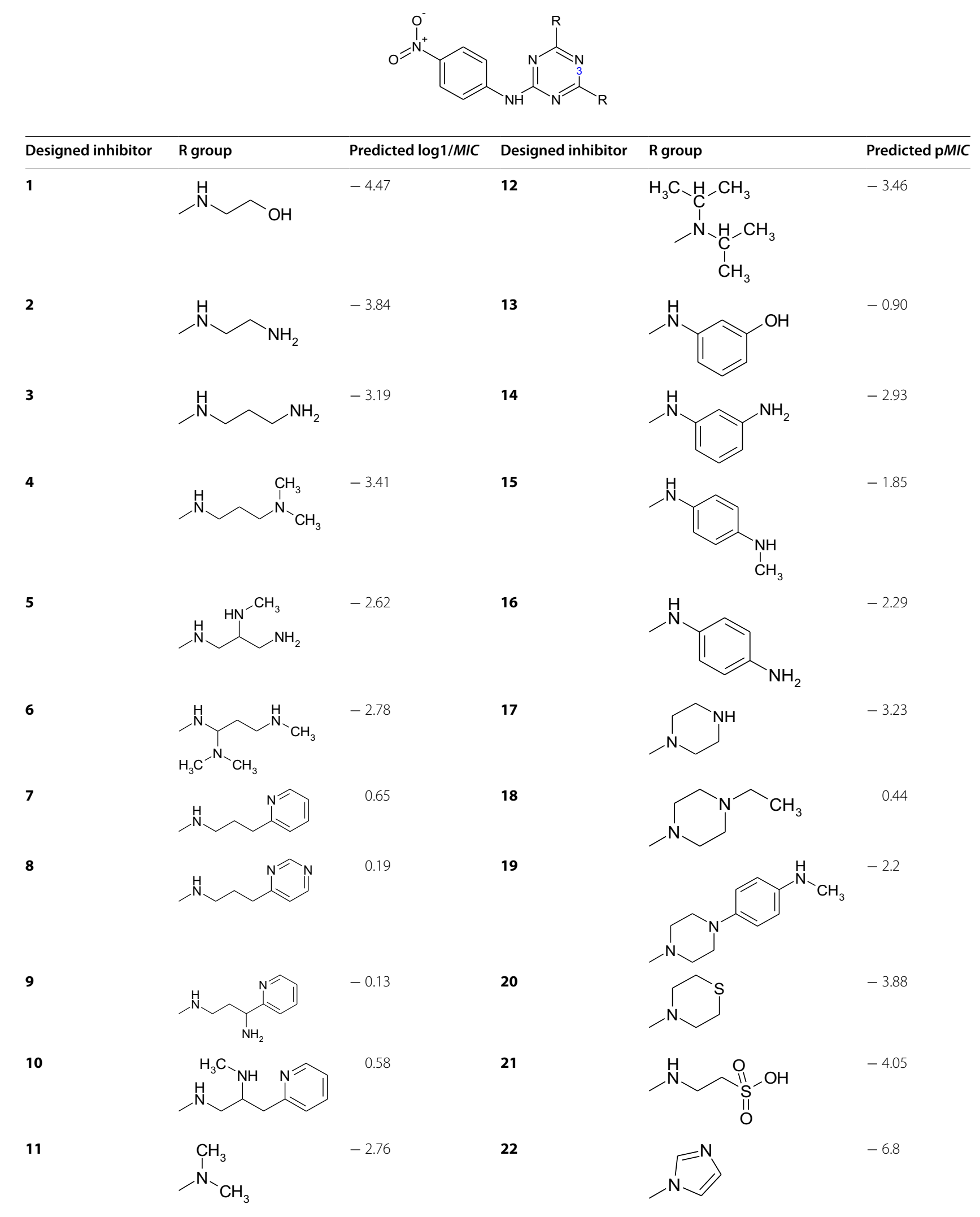


The program was processed in the normal mode, and 44 properties were predicted for the $22 s$-triazine compounds. These predicted properties consist of principal descriptors and physicochemical properties with a detailed analysis of the octanol/water partition coefficient (QlogPo/w), octanol/gas partition coefficient (QlogPoct), water/gas partition coefficient $(\mathrm{Q} \log \mathrm{Pw})$, polarizability in cubic $\mathrm{A}^{0}$ (QPolrz), \% human absorption in the intestines $(\mathrm{QP} \%)$, brain/blood partition coefficient (QPlogBB), $\mathrm{IC}_{50}$ value of HERG $\mathrm{K}^{+}$blockage channels (logHERG), skin permeability (QPlogKp), binding to human serum albumin (QPlogKhsa), apparent Caco-2 cell permeability in $\mathrm{mm} / \mathrm{s}$ (QPPCaco), and apparent MDCK cell permeability in $\mathrm{mm} / \mathrm{s}$ (QPPMDCK). Caco-2 cell line is good model for the gut-blood barrier, while MDCK cell line is considered a good model for the blood-brain barrier. Besides, QikProp evaluates the acceptability of the compounds based on Lipinski's rule of five [29], which is essential for rational drug design. Low permeability and/or poor absorption for compounds results when a compound violates one or more than one Lipinski's rule of five (i.e. more than 5 hydrogen donors, the molecular weight is over 500 , the $\log \mathrm{P}$ is over 5 and the sum of N's and O's is over 10).

\section{Chemistry \\ General procedure for the synthesis of compounds $(7,13$ and 18)}

The 2,4,6-trisubstituted-1,3,5-triazine compounds were synthesized by refluxing 2,4,6-trichloro-1,3,5-triazine (cyanuric chloride) with different nucleophiles (R). The mono-substituted triazine (4,6-dichloro- $N$-(4nitrophenyl)-1,3,5-triazin-2-amine) was synthesized by refluxing cyanuric chloride with $p$-nitroaniline in the presence of potassium carbonate in tetrahydrofuran (THF).

$N$-2-(4-Nitrophenyl)-N-4,N-6-bis[3-(pyridin-2-yl) propyl]-1,3,5-triazine-2,4,6-triamine (7) Yellow (solid). Yield 96\%. Mp: $144-1461{ }^{\circ} \mathrm{C}$; IR $\left(\mathrm{KBr}, \mathrm{v}_{\max }\right.$ in $\left.\mathrm{cm}^{-1}\right): 3412$ (N-H, str.); $1643\left(\mathrm{C}=\mathrm{N}\right.$, str.); 1537, $1372\left(\mathrm{NO}_{2}\right.$, str.); ${ }^{1} \mathrm{H}-$ NMR (400 MHz, $\left.\mathrm{CDCl}_{3}\right): 8.1-6.3(\mathrm{~m}, 12 \mathrm{H}, \mathrm{Ar}-\mathrm{H}) ; 4.5(\mathrm{~m}$, $\left.4 \mathrm{H}, \mathrm{CH}_{2}\right) ; 3.5\left(\mathrm{t}, 4 \mathrm{H}, \mathrm{CH}_{2}\right) ; 2.8\left(\mathrm{t}, 4 \mathrm{H}, \mathrm{CH}_{2}\right) ; 5.6(\mathrm{~s}, 1 \mathrm{H}, \mathrm{NH})$; ${ }^{13} \mathrm{C}-\mathrm{NMR}\left(100 \mathrm{MHz}, \mathrm{CDCl}_{3}\right.$ ) $\delta$, ppm: 172.8, 169.5, 164.3, 154.2, 145.3, 141.9, 137.6, 131.2, 127.2, 113, 100.9, 79.0, 57.9, 54.6, 39.9; Anal. Calcd. for $\mathrm{C}_{25} \mathrm{H}_{27} \mathrm{~N}_{9} \mathrm{O}_{2}$ C: 61.62; $\mathrm{H}$ : $5.25 ; \mathrm{N}: 24.71$; found: C: $61.50 ; \mathrm{H}: 5.91 ; \mathrm{N}: 25.86$. Mass spectrum (ESI) $(\mathrm{M}+\mathrm{H})^{+}=486.6$

3-[4-(3-Hydroxyphenylamino)-6-(4-nitrophenylamino)-1,3,5-triazin-2-ylaminolphenol (13) Black (solid). Yield 98\%. Mp: $180-182^{\circ} \mathrm{C}$; IR $\left(\mathrm{KBr}, \mathrm{v}_{\max }\right.$ in $\left.\mathrm{cm}^{-1}\right)$ : 3409 (OH, str.); 1631 (C=N, str.); 1591, $1326\left(\mathrm{NO}_{2}\right.$, str.); 1180 (C-O, str.); ${ }^{1} \mathrm{H}-\mathrm{NMR}\left(400 \mathrm{MHz}, \mathrm{DMSO}-d_{6}\right)$ : 9.1-7.4 (m, 12H, Ar-H); 5.9 (s, 3H, NH); ${ }^{13} \mathrm{C}$ NMR $(100 \mathrm{MHz}$, DMSO- $\left.d_{6}\right) \delta$ ppm: $169,167.4,152,135.4,131.7,126.7$, 123, 120.9, 119.3, 117.3; Anal. Calcd. for $\mathrm{C}_{21} \mathrm{H}_{17} \mathrm{~N}_{7} \mathrm{O}_{4} \mathrm{C}$ : 58.47; H: 3.97; N: 22.73; found: C: 58.53; H: 4.01; N: 22.64 . Mass spectrum (ESI) $(\mathrm{M}+\mathrm{H})^{+}=432.1$

4,6-bis(4-Ethylpiperazin-1-yl)-N-(4-nitrophenyl)-1,3,5-triazin-2-amine (18) White (solid). Yield 97.5\%. Mp: 160$161{ }^{\circ} \mathrm{C}$; IR (KBr, $v_{\max }$ in $\left.\mathrm{cm}^{-1}\right) 3293(\mathrm{~N}-\mathrm{H}, \mathrm{str}) ; 1599,1660$ $\left(\mathrm{C}=\mathrm{N}\right.$, str.); 1541, $1317\left(\mathrm{NO}_{2}\right.$, str.); ${ }^{1} \mathrm{H}-\mathrm{NMR}(400 \mathrm{MHz}$, $\left.\mathrm{CDCl}_{3}\right)$ : 7.6-7.1 (m, $\left.4 \mathrm{H}, \mathrm{Ar}-\mathrm{H}\right) ; 3.9\left(\mathrm{q}, 4 \mathrm{H}, \mathrm{CH}_{2}\right) ; 3.5(\mathrm{~s}$, $1 \mathrm{H}, \mathrm{NH}) ; 3.28\left(\mathrm{t}, 4 \mathrm{H}, \mathrm{CH}_{2}\right) ; 2.9\left(\mathrm{t}, 6 \mathrm{H}, \mathrm{CH}_{3}\right) ;{ }^{13} \mathrm{C} \mathrm{NMR}$ $\left(100 \mathrm{MHz}, \mathrm{CDCl}_{3}\right) \delta$ ppm: 172.1, 155.3, 141.8, 135.7, 130.4, 114.3, 82, 77.3, 55.6, 43.5, 37.6; Anal. Calcd. for $\mathrm{C}_{21} \mathrm{H}_{31} \mathrm{~N}_{9} \mathrm{O}_{2}$ C: 57.13; H: 7.08; N: 28.55; found: C: 57.07; H: 7.11; N: 28.52. Mass spectrum (ESI) $(\mathrm{M}+\mathrm{H})^{+}=442.9$

\section{Pharmacology \\ Plasmodium parasite culture}

Stock culture of malaria parasite Plasmodium falciparum 3D7 strain was continuously maintained in vitro using the candle-jar method [30]. The Plasmodium falciparum 3D7 strain was maintained on $\mathrm{B}^{+}$human red blood cells. The aqueous culture media $(960 \mathrm{~mL})$ consisted of $10.4 \mathrm{~g}$ of RPMI- 1640 with $40 \mathrm{mg}$ of gentamicin and $5.94 \mathrm{~g}$ of HEPES buffer. The culture medium was reconstituted just before use by pouring sterile $5 \%$ sodium bicarbonate in ratio of 1:24 and the culture was further supplemented with $10 \%$ Bovineserum. The parasitemia culture was maintained in between 1 and $5 \%$ and routinely subculturing was performed on every fourth day. The hematocrit was maintained initially at $7 \%$.

\section{Plasmodium dilutions preparation}

Each compound was dissolved separately in DMSO to obtain stock solutions of $1 \mathrm{mg} / \mathrm{mL}$ concentration. The graded concentration of each compound used was as follows: $10,5,2,1$, and $0.1 \mu \mathrm{g} / \mathrm{mL}$. The working solutions of the desired concentration were prepared freshly by diluting the stock solutions of compounds. The final concentration of DMSO used in the culture media did not affect the parasite growth.

\section{Inhibitory concentration assay}

The minimum inhibitory concentrations of each compound were determined in vitro using a dose-response assay in 24-well tissue culture plates in triplicates. Synchronous parasites were prepared [31] to obtain parasitized cells harbouring only the ring stage and challenged with a graded concentration ranging from 0.1 to $10 \mu \mathrm{g} / \mathrm{mL}$ of the drug solution for $48 \mathrm{~h}$ at $37^{\circ} \mathrm{C}$ by the candle-jar method [30]. The medium was changed 
routinely after $24 \mathrm{~h}$ in each of wells (with or without the drug). Thin smear with Giemsa-staining were prepared and analyzed to determine the percentage inhibition of parasitemia vis-a-vis the control.

\section{Plasmodium slide preparation}

The 96-well plates were taken out from the candle jar and the material from each well was transferred into the corresponding well labelled $1.5-\mathrm{mL}$ microcentrifuge tube. After vortexing, the supernatant was pipetted out and the pellet was further spread thoroughly on a slide to prepare a thin blood smear slide for each well. Subsequently the smeared slides were air-dried, fixed with methanol and stained with Giemsa dye for $40 \mathrm{~min}$. After staining, the excess dye was removed by washing the slides in running tap water and finally slides were again air-dried. The stained slides were examined in random adjacent microscopic fields to count the number of parasites equivalent to approximately 3000 erythrocytes at $100 \times$ magnification.

\section{Results and discussion Design of new inhibitors}

In the QSAR model, the following properties appear in the top-most equations: $\chi(3)$ cluster, $k(1)$, Wiener index, Coulson charge on $\mathrm{N}_{3}$, Electrostatic charge on $\mathrm{C}_{2}$, Dipole moment (x), Total dipole, Octupole moment and Total energy. This list indicates that structural (topological) as well as electronic factors contribute to the activity or inactivity of a given compound. However, we require a deeper introspection of the actual quantitative effect of these parameters on the activity value. Deciphering the information available from a QSAR model needs the study of coefficients of these properties as they appear in the top equations.

The most powerful factor here is the charge on the nitrogen atom of the triazine ring, sandwiched between the two side chains. This indicates that the electron density at the triazine group should not decrease. So it was rational to attach electron donating atoms in these side chains.

$X(3)$ cluster contributes negatively towards activity. It leads us to keep less clustering in the side chains. $\kappa(1)$ has a positive coefficient, though of a comparatively lower value. It signifies that contacts of first degree between atoms are beneficial in improving the activity or we can say that branching is not a favourable trait. Clustering could result in bad grades. Very long chains are also not recommended as elongation of the side chain has no major effect on the electronic contribution towards activity. These points motivated us to choose simple $2-3$ carbon atom chains to be introduced near the triazine moiety.
Considering the factors described above, a series of $\mathrm{R}$ groups were attached to the triazine ring. Structural features of the compounds so obtained are given in Table 1, along with the predicted $\mathrm{p} M I C$ values, based on the first QSAR equation, for the corresponding derivatives.

$$
\begin{aligned}
\mathrm{Y}= & 0.2387(1)-2.2704(3)-0.3014 \mu_{\mathrm{T}} \\
& -0.2207 \mu_{\mathrm{x}}-00.7935 \mathrm{q}_{\mathrm{c}}-37.4695
\end{aligned}
$$

where, $\kappa(1)$ is the shape descriptor, $\chi(3)$ is the molecular connectivity indices, $\mu_{\mathrm{T}}$ is the total dipole moment, $\mu_{\mathrm{x}}$ is the dipole moment in the $\mathrm{X}$ direction and $\mathrm{q}_{\mathrm{c}}$ is the coulson charge on nitrogen atom.

It can be seen that substitution of electron donating functional groups at various positions lead to an increase in the activity of the derivatives. It is clear that attachment of an electron acceptor decreases the predicted value. An alcoholic group reduces $\mathrm{p} M I C$ to -4.47 (compound 1). Replacement of $-\mathrm{OH}$ with $-\mathrm{NH}_{2}$ improves the activity to a small extent (compound 2). Therefore various kinds of groups, such as phenyl, heterocyclic aromatic and aliphatic 5-6 member rings, and small aliphatic chains, were taken and the $-\mathrm{NH}_{2}$ group was added at different positions on the chain and at rings so as to get higher pMIC values (example-compounds 3, 9). Addition of methylamine and ethylamine proved to be better than amine (e.g. compare the pairs compounds 5 and 6; 15 and 16). Elongation of chain length also results in slightly better activity. An additional methyl group in the chain causes a slight increment in the biological activity. This can be seen as we move from compound 2-3. However, clustering and branching of any kind is not at all beneficial. Whenever an isopropyl or isobutyl group is added instead of an ethyl or methyl group, the activity for the resulting compound decreases (as in case of $\mathbf{4 , 1 1}$ and 12). In this course of action, we obtained new compounds which had better value than the existing compounds used in the QSAR study. Based on the overall analysis we can conclude that the compounds 7, 8, 9, 10, 13 and 18 (with $\mathrm{p} M I C: 0.65,0.19,-0.13,0.58,-0.90$ and 0.44 , respectively) are the most potent derivatives that could prove to be better drugs than the existing ones.

\section{ADME analysis and molecular docking}

In ADME screening, 44 parameters were calculated, which included molecular descriptors and pharmaceutically relevant properties like the partition coefficient $(\log \mathrm{Po} / \mathrm{w})$ and water solubility $(\log \mathrm{S})$, critical for estimation of absorption and distribution of drugs within the body, the blood brain barrier permeability $(\log B B)$ which is prerequisite for the entry of drugs to the brain, (log $\mathrm{Kp})$ predicted skin permeability, (logKhsa) prediction of binding to human serum albumin, $\left(\mathrm{P}_{\text {caco }}\right)$ model for the 
gut-blood barrier, percentage of human oral absorption and Lipinski's rule of five was considered a parameter to screen the best candidates out of 22 compounds.

It was observed that out of 22 compounds only 6 lead compounds were found not violating any of ADME property while the rest of 16 designed compounds have violated few important ADME properties like $\mathrm{P}_{\text {caco }}$, which is predicted apparent Caco- 2 cell permeability in $\mathrm{nm} / \mathrm{s}$ and are good model for gut-blood barrier, $\operatorname{logKhsa}$ that is the prediction of binding of ligand to the human serum albumin which in turn influence the biodistribution of drug in the blood and Lipinski's Rule. From Table 2, it was observed that all 6 lead compounds also have percentage human oral absorption more than $50 \%$ and allowed logKhsa values. Therefore, it is suggested that good binding ability with human serum albumin and reasonably good oral human absorption may result into better distribution and good absorption of these lead compounds. $\mathrm{P}_{\text {caco }}$ values suggested that these lead compounds may result into good absorption of these compounds through intestine, which is must for good absorption of a drug through oral route. However, additionally the docking study was performed to predict how the designed potential antimalarial compounds will bind to putative receptor (DHFR). The binding ability of ligands to receptor protein was determined on the basis of glide score found out by molecular docking method performed by method given in Additional file 1.

Table 2 displayed that all 6 hits have diverse glide scores ranging from -6.230 to -4.254 which were higher visa-vis that of cycloguanil standard antimalarial drug that works through this pathway. The 2-dimensional interaction maps suggested that in the docking site of DHFR, both hydrophobic interactions and hydrogen bonding were the dominant forces. It is well known through various published works and our own experience that hydrophobic and hydrogen binding interaction play pivotal role in complexation of ligands with proteins [32,33].

Therefore, from the comparison of compounds selected on the basis of predicted MIC values, docking score and ADME analysis respectively, compound no 11, 15, 20 were ruled out of the 6 compounds selected on the basis of predicted $p M I C$ values. However, we tried to synthesize all 6 lead compounds, but due to practical problems it was not possible to synthesize all the selected compounds. Compounds 7, 13 and 18 are the only three compounds which could be synthesized.

Figures 1, 2 and 3 showed the 3-dimensional docked models for compounds 18, 7 and $\mathbf{1 3}$ in the binding site of receptor protein DHFR. Compound $\mathbf{1 8}$ when docked in the binding site (Fig. 1) formed the hydrogen bond with LYS 359 involving oxygen atom of the nitro group,

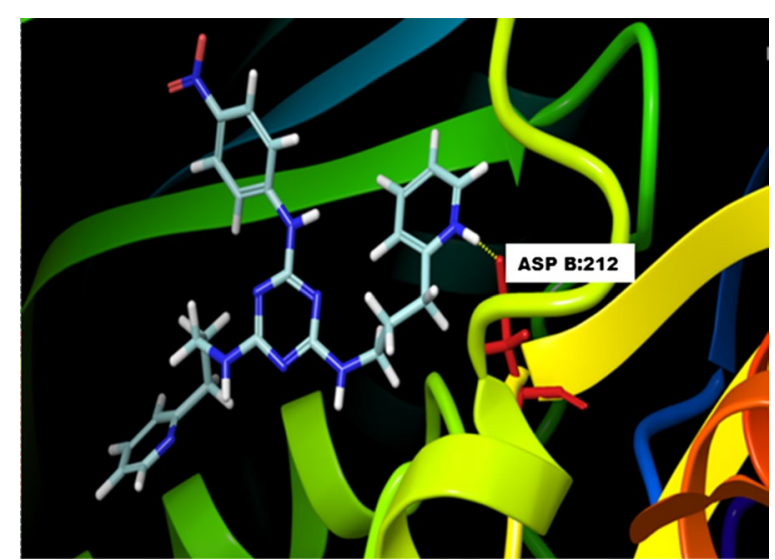

Fig. 1 3-D docked for binding of compound no $\mathbf{1 8}$ in the active site of DHFR

Table 2 The ADME properties and Glide score of the selected six lead candidates

\begin{tabular}{|c|c|c|c|c|c|c|c|c|}
\hline Lead compounds & $\log \mathrm{Po} / \mathrm{W}^{\mathrm{a}}$ & $\log S^{b}$ & PCaco $^{c}$ & loghsad & $\log B B$ & $\log K p$ & $\begin{array}{l}\text { \% human oral } \\
\text { absorption }^{\mathrm{e}}\end{array}$ & $\begin{array}{l}\text { Glide score } \\
\text { for inhibition } \\
\text { of Pf-DHFR } \\
\text { (Kcal/mol) }\end{array}$ \\
\hline 13 & 2.421 & -5.30 & 27.88 & 0.09 & -3.01 & -3.93 & 54.03 & -6.23 \\
\hline 20 & 3.841 & -6.17 & 551.04 & 0.58 & -0.72 & -3.15 & 100.00 & -5.41 \\
\hline 7 & 4.614 & -6.39 & 184.56 & 0.59 & -2.30 & -2.05 & 81.56 & -5.25 \\
\hline 15 & 3.333 & -6.15 & 100.16 & 0.31 & -2.42 & -3.06 & 69.31 & -4.74 \\
\hline 11 & 2.778 & -4.19 & 619.07 & 0.05 & -0.97 & -2.81 & 93.17 & -4.64 \\
\hline 18 & 2.409 & -3.75 & 37.21 & 0.33 & -0.36 & -7.04 & 96.13 & -4.25 \\
\hline Cycloguanil & - & - & - & - & - & - & - & -4.17 \\
\hline
\end{tabular}

\footnotetext{
${ }^{a} \log \mathrm{Po} / \mathrm{w}(-2.0$ to 6.5$)$

b $\log S$ ( -6.5 to 0.5 )

c Pcaco $<25$ is very poor and $<500$ is great

d logkhsa ( -1.5 to 1.5$)$

e $\%$ human oral absorption ( $<25 \%$ is poor and $>80 \%$ is high)
} 


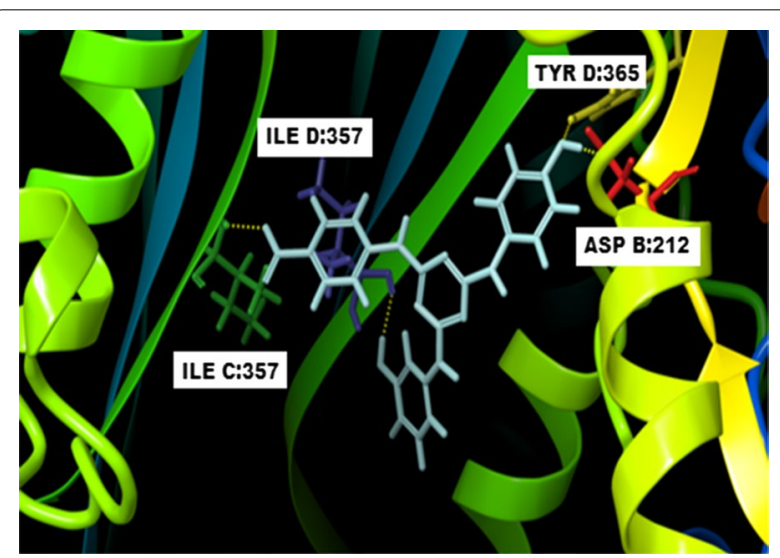

Fig. 2 3-D docked for binding of compound no $\mathbf{7}$ in the active site of DHFR

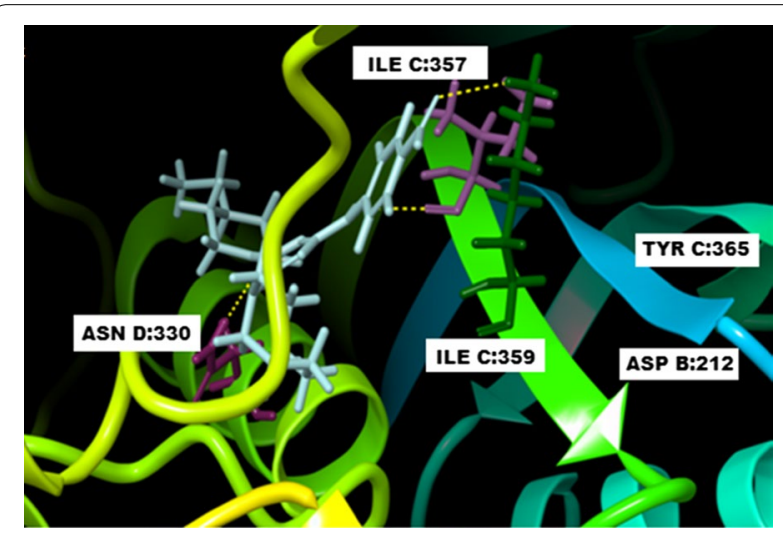

Fig. 3 3-D docked for binding of compound no $\mathbf{1 3}$ in the active site of DHFR

sandwiched - NH- group with ILE357 and between one of ring nitrogen atoms of triazine ring and ASN330 of the receptor. While the two dimensional interaction map (Additional file 1: Figure S2a) indicated that compound 18 maintained hydrophobic interactions with seven interacting residues in the binding site. Besides, $\mathrm{NH}^{+}$ group of piperazine ring formed charged transition with ASP334. Similarly, one of the charged oxygen atom of nitro group formed salt bridge with LYS359.

In compound 7, there is preponderance of hydrophobic interactions with 11 residues in the binding site (Fig. 2 and Additional file 1: Figure S2b). There was single hydrogen bond formation between nitrogen atom of 2-pyridine and ASP212 of the binding site. Further, on comparing the 2-dimensional interaction maps of compounds 18 and 7, it was observed that two residues ILE357 and TYR356 of C chain of DHFR were found common in the binding site which formed hydrophobic interactions. Similar to compound 18, nitro group of the backbone of ligand formed salt bridge with LYS359.

During the docking of compound 13 with Plasmodium falciparum DHFR, it was observed that there was formation of four hydrogen bonds between the ligand and four interaction residues of the binding site. One of the phenol group formed hydrogen bond involving oxygen atom ILE357 ( $\mathrm{D}$ chain) where oxygen atom act as donor and on the contrary, oxygen atom of other substituted phenol ring formed two hydrogen bonds separately with TYR365 and ASP212 respectively and act as hydrogen bond acceptor. The fourth hydrogen bond was formed between $\mathrm{O}$ atom of nitro group and residue of chain C ILE357 (Fig. 3 and Additional file 1: Figure S2c). On comparing the two dimensional interaction maps of all three compounds two residues ILE357 and TYR356 were found common for hydrophobic interaction. While there was more than $80 \%$ commonness in the interacting residues on the binding sites which play an important role in hydrophobic interactions for compounds 7 and 13. Therefore, close examination of compounds 7 and $\mathbf{1 3}$ suggested that hydrophobic interaction play an equal role for both compounds but compound $\mathbf{1 3}$ has more hydrogen bond formation and highest potency.

\section{Chemistry}

The compounds 7, 13 and 18 were synthesized by refluxing 2,4,6-trichloro-1,3,5-triazine (cyanuric chloride) with different nucleophiles (R) (Scheme 1). The three compounds were synthesised by some modifications in the literature procedure, which exploits a nucleophilic substitution reaction under solid-liquid phase-transfer conditions. The reaction, which is carried out in the presence of $\mathrm{K}_{2} \mathrm{CO}_{3}$ and a catalytic amount of 18-crown6, allows possibility to substitute, by employing the appropriate number of equivalents of the nucleophile and $\mathrm{K}_{2} \mathrm{CO}_{3}$, one, two, or all of the chlorine atoms of 2,4,6-trichloro-1,3,5-triazine, only by appropriate reaction temperature. Normally the first substitution in cyanuric chloride takes place at $0{ }^{\circ} \mathrm{C}$, but the amino group of $p$-nitroaniline is highly deactivated due to the presence of a nitro group at the para position (Scheme 1 and synthetic scheme in Additional file 1). All the synthesized compounds were characterized by their spectroscopic data, such as IR, NMR, Mass Spectrometry and elemental analysis. The synthesized trisubstituted triazine compounds are shown in Table 3.

\section{Spectroscopic characterization}

The skeleton of all three final products (2,4,6-s-triazines) have been identified by mass spectrum. The mass spectrum of compound 7 showed a molecular ion peak at $m / z=486.6$ amu corresponding to $(\mathrm{M}+\mathrm{H})^{+}$, which 


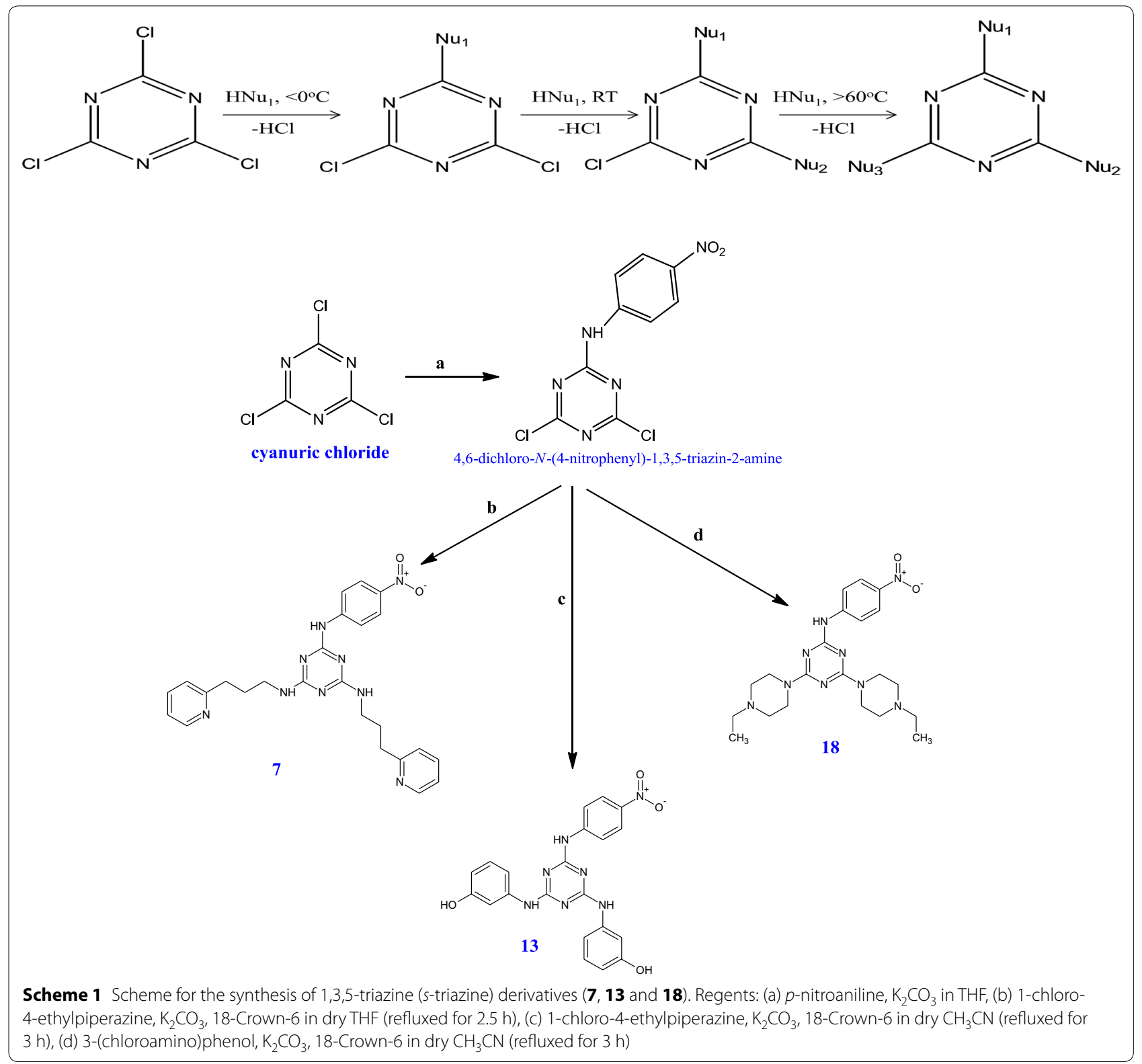

confirms the proposed formula $\left(\mathrm{C}_{25} \mathrm{H}_{28} \mathrm{~N}_{9} \mathrm{O}_{2}\right)^{+}$and its base peak was observed at $m / z$ 363.2. Similarly, the molecular ion peak of compound $\mathbf{1 3}$ was found at 432.1 and for compound $\mathbf{1 8}$ at 442.9 (Additional file 1: Figure S3).

The IR spectra of the compound 7 (Additional file 1: Figure S4) shows a high intensity band at $3412 \mathrm{~cm}^{-1}$ due to the presence of intramolecular hydrogen bonding because of the presence of three $\mathrm{NH}$ groups. The absorption bands at 1537 and $1372 \mathrm{~cm}^{-1}$ are due to $\mathrm{NO}_{2}$ stretching vibrations. The IR bands at 1643, 1269, 2841 and $968 \mathrm{~cm}^{-1}$ are assigned to $\mathrm{C}=\mathrm{N}, \mathrm{C}-\mathrm{N}, \mathrm{CH}_{2}$ and $\mathrm{N}-\mathrm{O}$ stretching vibrations, respectively. The IR peak at $730 \mathrm{~cm}^{-1}$ corresponds to a substituted pyridine ring, which further establishes the structure of compound 7. Similarly, Additional file 1: Figure S5 showed the IR spectrum of the compound $\mathbf{1 3}$ displayed the overlapped region of $\mathrm{OH}$ and $\mathrm{NH}$ stretching bands in the $3200-3400 \mathrm{~cm}^{-1}$ region due to the presence of two terminal phenolic groups and three $\mathrm{NH}$ groups which are symmetrically attached to the triazine ring. The IR peak at $3409 \mathrm{~cm}^{-1}$ corresponds to the $\mathrm{O}-\mathrm{H}$ stretching vibration. The IR bands at 1631, 1261, 3081 and $1180 \mathrm{~cm}^{-1}$ are assigned to $\mathrm{C}=\mathrm{N}, \mathrm{C}-\mathrm{N}, \mathrm{Ar}-\mathrm{H}$ and $\mathrm{C}-\mathrm{O}$ stretching vibrations, respectively. Also, the presence of the $\mathrm{NO}_{2}$ group was confirmed by the presence of two IR stretching bands at 1591 and $1326 \mathrm{~cm}^{-1}$. 


\section{Table 3 Synthesized trisubstituted triazine compounds}

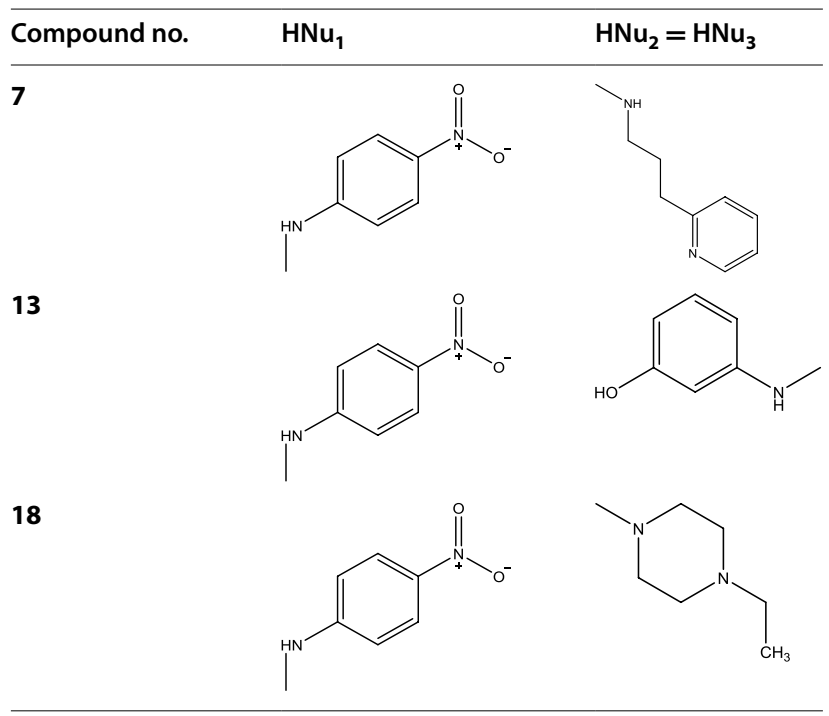

The IR spectrum of compound $\mathbf{1 8}$ (Additional file 1: Figure S6) indicated the absorption band at $3293 \mathrm{~cm}^{-1}$ is related to the $\mathrm{N}-\mathrm{H}$ stretching vibration. The absorption bands at 1559 and $1660 \mathrm{~cm}^{-1}$ are due to $\mathrm{C}=\mathrm{N}$ stretching vibrations, confirming the presence of triazine rings in the compound; the IR bands at 1541 and $1317 \mathrm{~cm}^{-1}$ are assigned to $\mathrm{NO}_{2}$ stretching vibrations. The presence of the $\mathrm{NO}_{2}$ group in the compound is further confirmed by the stretching vibration peak at $966 \mathrm{~cm}^{-1}$ corresponding to the $\mathrm{N}-\mathrm{O}$ stretching vibration. The structure of compound 18 was further confirmed by the presence of absorption bands at 1257, 2845 and $1365 \mathrm{~cm}^{-1}$ due to $\mathrm{C}-\mathrm{N}, \mathrm{CH}_{2}$ and tertiary $\mathrm{N}$, respectively.

The ${ }^{1} \mathrm{H}$-NMR spectra have been recorded for compounds 7, 13 and 18. In compound 7, 6 aliphatic methylene groups were found in the region of $\delta 2.8-4.5 \mathrm{ppm}$. The 12 aromatic protons were confirmed by the integral of the ${ }^{1} \mathrm{H}$-NMR spectrum and were found to lie in the region of $\delta 6.3-8.1 \mathrm{ppm}$. The three $\mathrm{NH}$ groups were confirmed by a singlet at $\delta 5.6 \mathrm{ppm}$ in the ${ }^{1} \mathrm{H}$-NMR spectrum of compound 7 (Additional file 1: Figure S7). The ${ }^{1} \mathrm{H}$ NMR spectra of compound 13 (Additional file 1: Figure S8) confirmed the presence of twelve aromatic protons in the region of $\delta 7.4-8.9 \mathrm{ppm}$. The spectrum showed a singlet at $\delta 9.16 \mathrm{ppm}$, which confirmed the presence of phenolic protons in the compound. Three different labile protons, belonging to three $\mathrm{NH}$ groups, were found as a singlet at $\delta 5.9 \mathrm{ppm}$, which further confirmed the structure of compound 13.

Similarly, the ${ }^{1} \mathrm{H}$-NMR spectra of compound 18 (Additional file 1: Figure S9), confirms the presence of three chemically non-equivalent methylene proton groups at

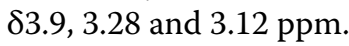

Table 4 MIC values of the synthesized 2,4,6-trisubstituted-1,3,5-triazine derivatives against 3D7 strain of $P$. falciparum

\begin{tabular}{ll}
\hline Compound & Minimum inhibitory concentration $^{\mathbf{a}}$ \\
\hline $\mathbf{7}$ & 4.466 \\
$\mathbf{1 3}$ & 7.94 \\
$\mathbf{1 8}$ & 2.75 \\
Cycloguanil & 255 \\
\hline
\end{tabular}

${ }^{a}$ Minimum inhibitory concentration for the development of ring stage parasite into the schizont stage during $48 \mathrm{~h}$ incubation

The ${ }^{1} \mathrm{H}$-NMR signal at $\delta 3.5 \mathrm{ppm}$ was caused due to $\mathrm{D}_{2} \mathrm{O}$ exchange and confirms the presence of a labile proton attached to the nitrogen, as the $\mathrm{NH}$ group is sandwiched between the two aromatic rings in the compound. The multiplet in the region of $\delta 7.1-7.6 \mathrm{ppm}$ confirms the presence of four aromatic protons. $\mathrm{N}$ terminal aliphatic methyl protons were also confirmed by its deshielded lower $\delta$ values.

The ${ }^{13} \mathrm{C}$-NMR spectra was recorded for three compounds; 7, 13 and 18. In compound 7, there were 6 aliphatic methylene groups were found in the region of $\delta$ 39.9-64.9 ppm and 19 aromatic carbons in the region of $\delta 79-172.8 \mathrm{ppm}$ (Additional file 1: Figure S10). The ${ }^{13} \mathrm{C}$-NMR spectrum of compound $\mathbf{1 3}$ has showed 21 peaks of aromatic carbons falling in the region of $\delta$ 117.7-169 ppm (Additional file 1: Figure S11). In ${ }^{13} \mathrm{C}$ NMR spectrum of compound 18, 12 aliphatic methylene groups were found in the region of $\delta 29.5-82 \mathrm{ppm}$ and 9 aromatic carbons in the region of $\delta 101.4-172.1 \mathrm{ppm}$ (Additional file 1: Figure S12).

\section{Antimalarial activity evaluation}

All the prepared compounds 7, 13 and 18 were found to be active against the 3D7 strain (cycloguanil sensitive strain) of Plasmodium falciparum species and all the analogues show minimum inhibitory concentrations (MIC) in the low nano molar range from 2.75 to $7.94 \mu \mathrm{mol} / \mathrm{L}$ (Table 4).

The MIC values of the synthesized triazine derivatives were compared with the reported MIC value of cycolguanil, which is taken as a standard drug under the study. The MIC value of cycloguanil has been reported as $64 \mu \mathrm{g} /$ $\mathrm{mL}(\sim 255 \mathrm{~nm})$ against the NF54 strain of Plasmodium falciparum species. Since 3D7 is a drug sensitive laboratory clone of the NF54 isolate and both of them are closely related to each other [34], the reported MIC value of $64 \mu \mathrm{g} /$ $\mathrm{mL}$ was considered reference MIC for comparison with the MIC values of prepared $s$-triazine analogues against the 3D7 strain of the Plasmodium falciparum species. It was found, from our activity data of the three synthesized triazine derivatives that all three synthesized compounds 
were more potent than cycloguanil and compound $\mathbf{1 8}$ is the most active out of the three compounds against the Plasmodium falciparum species, with an MIC value of $2.75 \mu \mathrm{mol} / \mathrm{L}$. Compound 7, with a propyl group linked to 2-pyridine in the substituent part, was found to be less effective against the parasite, than the compound 18, as its $\mathrm{MIC}$ value is $4.466 \mu \mathrm{mol} / \mathrm{L}$. Compound 13, was observed to be the least active against the parasite out of the three compounds, as it showed the highest MIC value of $7.94 \mu \mathrm{mol} / \mathrm{L}$.

This data revealed that the compound $\mathbf{1 3}$ with an aromatic ring (with electron donating group) in the substituent part contributed positively towards the antimalarial action. While the ring consisting of electron withdrawing group may cause an increase in the MIC value as showed by compound 7. Another important feature to correlate biological activity is the number of hydrogen bond formations. In the docking data it was discussed previously that in compound 13 there were four hydrogen bond formations while numbers decreased for compound $\mathbf{1 8}$ and least for compound 7. In the same order, the biological activity varies for the prepared compounds highlighted and established the importance of hydrogen bonding for DHFR inhibition.

Another important dimension in the discussion, which is worth mentioning, is the correlation of two physicochemical properties calculated for these derivatives with MIC values. The first is the LogPo/w values, which describe the lipophilicity of a drug. The calculated $\mathrm{LogPo} / \mathrm{w}$ values for the above-mentioned compounds 7, 13 and 18 are 4.61, 2.409 and 2.42, respectively. On matching these with the experimental MIC values of these compounds, it is found that the most potent compound is the least lipophilic one and the trend is that MIC follows those of the lipophilicity. This emphasizes the importance of lipophilicity in the antimalarial activity. The second useful physicochemical property is the coulson charge on the third nitrogen atom placed in the triazine ring of the basic structure of the compounds. On consideration of all five QSAR built models, it is found that out of the six important molecular descriptors, the descriptor which heavily contributes to the activity is the coulson charge of the nitrogen atom in triazine ring, which is sandwiched between two carbons atoms of same ring over which substitution were performed. The property, as obtained computationally, has values -0.3401 , -0.3555 and -0.3479 , respectively for the three synthesized compounds 7, 13 and $\mathbf{1 8}$. This property was found to be directly correlated to the MIC value of the compound and this is confirmed by our experimental results. The smallest value of the coulson charge, -0.3555 , was obtained for compound $\mathbf{1 3}$ and the least MIC value of $4.2 \pm 0.02 \mathrm{nM}$ is also found for the same compound. The other two compounds further confirm to the same trend. This validates the correlation between the structural features of the triazine analogues and their antiplasmodial activity against the Plasmodium falciparum species.

\section{Conclusion}

Therefore, in conclusion, the present methodology proved to be a facile and rapid procedure for the preparation of trisubstituted-1,3,5-triazine compounds. The compounds were designed based upon QSAR modelling and further screening was performed on designed inhibitors using ADME and docking studies. An attempt was made to synthesize six compounds, but practically three compounds were synthesized with significantly high yields in the range of $95-100 \%$ with high purity, as checked by thin layer chromatography (TLC). The triazine compounds were analysed satisfactorily, both by the spectral and analytical data. The IR, ${ }^{1} \mathrm{H}-\mathrm{NMR}$ and ${ }^{13} \mathrm{C}$ NMR data have been comprehensively discussed and complement each other for each compound. The triazine derivatives with desired structural features to promote requisite antimalarial property were shown to display desirable antimalarial activity and it was also confirmed using in silico investigations of synthesized inhibitors that hydrogen bonding, lipophilicity and coulson charge were found to correlate with the MIC values. The correlation was performed just to analyze the role of some important pharmacophoric features in DHFR inhibition.

\section{Additional file}

Additional file 1. Additional figures

\section{Authors' contributions}

MP carried the synthesis and characterization of compounds and helped in writing the manuscript; $\mathrm{HO}$ has designed and conducted spectroscopic characterization, analyzed the results and finalized the manuscript; AKT helped in availability of charaterization facilities and helped in in vitra study; DS has performed computational molecular docking studies; MS has performed QSAR and helped in molecular docking studies; RK has guided and did mentorship during the study and helped in finalizing the manuscript. All authors read and approved the final manuscript.

\section{Author details \\ ${ }^{1}$ Department of Chemistry, Miranda House, University of Delhi, Delhi 110007, India. ${ }^{2}$ Department of Chemistry, University of Delhi, Delhi 110007, India. ${ }^{3}$ Division of CBRN Defence, Institute of Nuclear Medicine and Allied Sciences, DRDO, Timarpur, Delhi 110054, India.}

\section{Acknowledgements}

Authors acknowledge encouragement of Director, INMAS and sincerely thank Head of Department of Chemistry, University of Delhi for providing equipment facilities support. One of the authors, Deepti Sharma is thankful to UGC for providing her junior research fellowship.

\section{Competing interests}

The authors declare that they have no competing interests.

Ethics approval and consent to participate

Not applicable. 


\section{Publisher's Note}

Springer Nature remains neutral with regard to jurisdictional claims in published maps and institutional affiliations.

Received: 3 October 2017 Accepted: 4 December 2017

Published online: 19 December 2017

\section{References}

1. WHO Global Malaria Programme (2015) World Malaria Report 2015. World Health Organization (WHO), pp 10-14

2. Operational Manual for Malaria Elimination in India 2016 (2016) National Vector Borne Disease Control Programme (NVBDCP) Directorate General of Health Services. Ministry of Health \& Family Welfare, Government of India, Delhi, pp 19-256

3. Milhous WK, Weatherly NF, Bowdre JH, Desjardins RE (1985) In vitro activities of and mechanisms of resistance to antifol antimalarial drugs. Antimicrob Agents Chemother 27:525-530

4. Crounse NN (1951) Isolation and identification of a metabolite of chlorguanide. J Org Chem 16:492-500

5. Crowther AF, Davey DG, Levi AA, Rose FL (1951) A metabolite of paludrine with high antimalarial activity. Nature 168:1080

6. Crowther AF, Levi AA (1953) Proguanil-the isolation of a metabolite with high antimalarial activity. Br J Pharmacol Chemother 8:93-97

7. Modest EJ (1956) Chemical and biological studies on 1, 2-dihydro-s-triazines. II. Three-component synthesis. J Org Chem 21:1-13

8. Modest EJ, Levine P (1956) Chemical and biological studies on 1,2-dihydro-s-triazines. III. Two-component synthesis. J Org Chem 21:14-20

9. Klenke B, Barrett MP, Brun R, Gilbert IH (2003) Antiplasmodial activity of a series of 1,3,5-triazine-substituted polyamines.J. Antimicrob Chemother 52:290-293

10. Agarwal A, Srivastava K, Puri SK, Chauhan PMS (2005) Synthesis of 2,4,6-trisubstituted triazine as antimalarial agents. Bioorg Med Chem Lett 15:531-533

11. Childs GE, Lambros C (1986) Analogues of N-benzyloxydihydrotriazines: in vitro antimalarial activity against Plasmodium falciparum. Ann Trop Med Parasitol 80:177-181

12. Rieckmann KH, Campbell GH, Sax LJ, Ema JE (1978) Drug sensitivity of Plasmodium falciparum. An in vitro microtechnique. Lancet 311:22-23

13. Gdaniec M, Saczewski F, Brzozowski Z (2003) 2-Amino-6-(1imidazolylmethyl)-4-(3,5,5-trimethyl-2-pyrazolin-1-yl)-1,3,5-triazine and 2-amino-6-(1-benzimidazolylmethyl)-4-(3,5,5-trimethyl-2-pyrazolin-1-yl)1,3,5-triazine hemihydrate. Acta Crystallogr C 59:696-699

14. Kreutzberger A, Richter B (1983) Antimycotic agents 13. Halophenoxy-1,3,5-triazines. Arch Pharm 316:213-218

15. Shabadi CV, Shelar MA, Shelar AR (1998) Synthesis of some new penicillin and cephalosporin derivatives of substituted s-triazine and their biological activity. Ind Drugs 35:488-493

16. Smolin EM, Rapoport L (1959) s-Triazine and derivatives in the chemistry of heterocyclic compounds. Interscience, New York

17. Patel RV, Kumari P, Rajani DP, Pannecouque C, De Clerca E, Chikhalia KH (2012) Antimicrobial, anti-TB, anticancer and anti-HIV evaluation of new s-triazine-based heterocycles. Future Med Chem 4:1053-1065
18. Shanmugam M, Narayanan K, Chidambaranathan V, Kabilan S (2013) Synthesis, spectral characterization and antimicrobial studies of novel s-triazine derivatives. Spectrochim Acta 105:383-390

19. Srinivas K, Srinivas U, Rao VJ, Bhanuprakash K, Kishore KH, Murty US (2005) Synthesis and antibacterial activity of 2,4,6-tri substituted s-triazines. Bioorg Med Chem Lett 15:1121-1123

20. Nishimura N, Kato A, Maeba I (2001) Synthesis of pyrrolo(2,1-f)(1,2,4) triazine C-nucleosides. Isosteres of sangivamycin, tubercidin, and toyocamycin. Carbohydr Res 331:77-82

21. Yakhontov LN, Vakhatova GM (1981) Search for medicinal preparations in the series of 1,3,5-triazines. Pharm Chem J 15:546-561

22. Chauhan PMS (2000) Currents and potential filaricides. Drugs Future 25:481-488

23. Chauhan PMS, Srivastava SK (2001) Present trends and future strategy in chemotherapy of malaria. Curr Med Chem 8:1535-1542

24. Blakley RL, Benkovic SJ (1984) Dihydrofolate reductase in folates and pteridines. Chemistry and biochemistry of folates: dihydrofolate reductase, vol 1. Wiley, New York, pp 191-251

25. Plowe CV, Cortese JF, Djimde A, Nwanyamwu OC, Watkins WM, Winstanley PA, Estrada-Franco JG, Mollinedo RE, Avila JC, Cespedes JL, Carter D, Doumbo OK (1997) Mutations in Plasmodium falciparum dihydrofolate reductase and dihydropteroate synthase and epidemiologic patterns of pyrimethamine-sulfadoxine use and resistance. J Infect Dis 176:1590-1596

26. Rastelli G, Sirawaraporn W, Sompornpisut P, Vilaivan T, Kamchonwongpaisan S, Quarrell R, Lowe G, Thebtaranonth Y, Yuthavong Y (2000) Interaction of pyrimethamine, cycloguanil, WR99210 and their analogues with Plasmodium falciparum dihydrofolate reductase: structural basis of antifolate resistance. Bioorg Med Chem 8:1117-1128

27. Ojha H, Gahlot P, Tiwari AK, Pathak M, Kakkar R (2011) Quantitative structure activity relationship study of 2,4,6-trisubstituted-s-triazine derivatives as antimalarial inhibitors of Plasmodium falciparum dihydrofolate reductase. Chem Biol Drug Des 77:57-62

28. Duffy EM, Jorgensen WL (2000) Prediction of properties from simulations: free energies of solvation in hexadecane, octanol, and water. J Am Chem Soc 122:2878-2888

29. Lipinski CA, Lombardo F, Dominy BW, Feeney PJ (2001) Experimental and computational approaches to estimate solubility and permeability in drug discovery and development settings. Adv Drug Deliv Rev 46:3-26

30. Trager W, Jensen JB (1976) Human malaria parasites in continuous culture. Science 193:673-675

31. Lambros C, Vanderberg JP (1979) Synchronization of Plasmodium falciparum erythrocytic stages in culture. J Parasitol 65:418-420

32. Ojha H, Mishra K, Hassan MI, Chaudhury NK (2012) Spectroscopic and isothermal titration calorimetry studies of binding interaction of ferulic acid with bovine serum albumin. Thermochim Acta 548:56-64

33. Ojha H, Murari BM, Anand S, Hassan Ml, Ahmad F, Chaudhury NK (2009) Interaction of DNA minor groove binder Hoechst 33258 with bovine serum albumin Chem Pharma Bull 57:481-486

34. Too K, Brown DM, Bongard E, Yardley V, Vivas L, Loakes D (2007) Antimalarial activity of N6-modified purine analogues. Bioorg Med Chem 15:5551-5562 